\title{
Mean platelet volume (MPV): new diagnostic indices for co-morbidity of tuberculosis and diabetes mellitus
}

\author{
Feifan $\mathrm{Xu}^{1,2+}$, Shengyan $\mathrm{Qu}^{2+}{ }^{+}$Lin Wang ${ }^{2}$ and Yongwei Qin ${ }^{1,3^{*}}$
}

\begin{abstract}
Background: Tuberculosis (TB) and type 2 diabetes mellitus (DM) are global health diseases with high morbidity and mortality. Few studies have focused on platelet indices in TB-DM coinfection patients. The objective of this work was to analyze the platelet indices in TB, DM and TB-DM patients to assess the predictive value of the platelet index for the risk of these diseases.
\end{abstract}

Methods: In total, 246 patients admitted to our hospital were distributed into three groups (113 TB, 59 DM and 74 TB + DM). A total of 133 individuals were also recruited as healthy controls (HC). Platelet indices, namely, platelet count (PC), mean platelet volume (MPV), plateletcrit (PCT) and platelet distribution width (PDW), were compared among the four groups, and the relationship with inflammatory markers was explored by using statistical software.

Results: Our study discovered that MPV and PCT were significantly downregulated in TB + DM patients $(9.95 \pm 1.25$ $\mathrm{fL}, 0.20 \pm 0.05 \%, P<0.0001, P=0.0121$, separately) compared with DM individuals (10.92 $\pm 1.17 \mathrm{fL}, 0.22 \pm 0.04 \%)$. Moreover, the changes in MPV were significantly higher in TB + DM patients $(9.95 \pm 1.25 \mathrm{fL}, P=0.0041)$ than in TB patients $(9.42 \pm 1.01 \mathrm{fL})$. No differences were found in PLT and PDW among the four groups $(P>0.05)$. The sensitivity and specificity of MPV in the differential diagnosis of DM patients vs TB + DM patients were 64.9 and 66.1\% $(P<0.0001)$, respectively, and the sensitivity and specificity of MPV between TB patients and TB + DM patients was 60.8 and $66.4 \%$, respectively $(P=0.003)$. MPV improved the diagnosis sensitivity when it was combined with clinical parameters, such as fasting blood glucose in DM and Mycobacterium tuberculosis culture result in TB $(76.3 \%$ vs $64.9,72.6 \%$ vs $60.8 \%, P<0.0001, P=0.001$, respectively). In addition, the sensitivity and specificity of $P C T$ in the differential diagnosis of DM patients vs TB + DM patients were 69.5 and $59.4 \%$, respectively $(P=0.008)$. PCT improved the diagnosis sensitivity when combined with fasting blood glucose in DM $(72.9 \%$ vs $64.9 \%, P=0.004)$. In addition, MPV was linked to CRP (C-reactive protein) and ESR (erythrocyte sedimentation rate) in the TB + DM patients $(r=0.3203, P=0.0054, r=0.2504, P=0.0307)$ but PCT was not $(r=0.1905, r=0.008675, P>0.05$, respectively). (Continued on next page)

\footnotetext{
* Correspondence: yw_qin@foxmail.com

There is increasing evidence that due to pathogenic mechanisms and metabolic factors, tuberculosis and diabetes have common risk factors that co-occur and cause mutual deterioration.

${ }^{\dagger}$ Feifan Xu and Shengyan Qu contributed equally to this work.

'Department of Pathogen Biology, School of Medicine, Nantong University,

19 Qixiu Road, Nantong 226001, Jiangsu, P.R. China

${ }^{3}$ Department of Cardiothoracic Surgery, Affiliated Hospital of Nantong

University, Nantong 226001, Jiangsu, P.R. China

Full list of author information is available at the end of the article
}

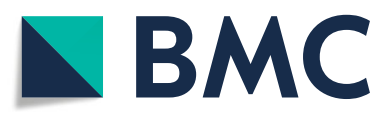

(c) The Author(s). 2021 Open Access This article is licensed under a Creative Commons Attribution 4.0 International License, which permits use, sharing, adaptation, distribution and reproduction in any medium or format, as long as you give appropriate credit to the original author(s) and the source, provide a link to the Creative Commons licence, and indicate if changes were made. The images or other third party material in this article are included in the article's Creative Commons licence, unless indicated otherwise in a credit line to the material. If material is not included in the article's Creative Commons licence and your intended use is not permitted by statutory regulation or exceeds the permitted use, you will need to obtain permission directly from the copyright holder. To view a copy of this licence, visit http://creativecommons.org/licenses/by/4.0/. The Creative Commons Public Domain Dedication waiver (http://creativecommons.org/publicdomain/zero/1.0/) applies to the data made available in this article, unless otherwise stated in a credit line to the data. 
(Continued from previous page)

Conclusions: Our research shows that MPV and PCT might be good clinical laboratory markers to distinguish TB + DM patients from TB or DM individuals, thus providing support for earlier clinical diagnosis, prevention, and therapy.

Keywords: Tuberculosis, Diabetes mellitus, Diagnosis indices, Mean platelet volume, Plateletcrit

\section{Background}

Tuberculosis (TB) is a global health disease caused by infection with Mycobacterium tuberculosis (MTB), despite its advanced developments in diagnosis and therapy [1]. In 2018, 10.0 million people were diagnosed with tuberculosis worldwide. China has the second largest tuberculosis epidemic worldwide, behind India, with more than 1.3 million new cases of tuberculosis every year. Ending the TB epidemic by 2030 is among the health targets of the Sustainable Development Goals.

Studies have demonstrated that some diseases accelerate TB occurrence and development [2]. Type 2 diabetes mellitus (DM) has been verified as one of the threatening risk factors for $\mathrm{TB}$, and patients have three times the risk of developing TB compared to nondiabetic patients due to pathogenic mechanisms and metabolic factors [3-5]. The DM prevalence among patients with TB in diversified low-income and middle-income countries ranged from 1.8 to $45 \%$, and TB prevalence among individuals with $\mathrm{DM}$ varied from 0.1 to $6.0 \%$ [6]. A national survey performed in 2010 found that the prevalence of DM was $11.2 \%$ (95\% confidence interval 10.5 to $11.9 \%$ ) in China [7]. Nearly $40 \%$ of TB cases in China and India are diabetes-related [8]. Clinically, DM facilitates TB development and hampers TB therapy, while conversely, TB impairs blood glucose control [9]. In addition, some studies have shown that proper blood glucose control will have a positive effect on reducing TB morbidity and mortality [10-12]. Published studies on the full prevalence of tuberculosis in Chinese patients with diabetes are far from sufficient. On the other hand, global evidence on the relationship between tuberculosis risk and blood glucose is still inconsistent [13-15]. To a great extent, TB and DM diagnoses are based on definitive detections, including clinical symptoms and characteristic $\mathrm{X}$-ray and laboratory examinations, with separate limitations [16]. Given the increasing global burden of DM, clinical and public health interventions against this coepidemic may lead to better tuberculosis prevention and treatment. However, due to the lack of established screening standards and methods, the effectiveness and reliability of screening for tuberculosis in patients with DM are not convincing [17]. In addition, few studies have focused on diagnostic markers to predict whether TB or DM will develop into a TB-DM coinfection (TB + DM). Thus, finding a feasible and cost-effective marker from laboratory reports for the early prevention and control of TB + DM is essential.

Platelets are enucleate cells and have critical roles in thrombosis, homeostasis and the inflammatory response [18]. When the internal environment is changed, platelet morphology may be altered and play a role in certain platelet-associated parameters, mainly including platelet count $(\mathrm{PC})$, plateletcrit $(\mathrm{PCT})$, platelet distribution width (PDW) and mean platelet volume (MPV). MPV detection and assays are generally valid in the clinic as they are routinely performed. MPV is increased in intestinal diseases, respiratory diseases, cardiovascular diseases, cerebral stroke, several cancers and diabetes. Conversely, MPV is decreased in ulcerative colitis, neoplasm, Systemic Lupus Erythematosus (SLE) and tuberculosis [19]. The mechanisms for an increased MPV are not well clarified. Several factors may influence MPV levels, including genetic variations, applied treatment drugs, lifestyle (diet, smoking, alcohol consumption, and physical activity), pre- and analytical procedures, hormonal profile, age, gender and race/ethnicity [19, 20]. Studies have demonstrated that changes in PC, especially during the process of $\mathrm{TB}$ infection, might be correlated with the mortality and severity of the infection [21, 22]. MPV is a marker reflecting the average size of platelets present in various diseases, such as $\mathrm{DM}$, metabolic syndrome and TB [23]. However, the role of MPV in TB is disputed. A study conducted by Gunluoglu et al. suggested that MPV, as an inflammation marker, decreased in active pulmonary tuberculosis related to the formation of microthrombi in TB cavities [24]. Conversely, Tozkoparan et al. suggested that MPV was significantly increased in patients suffering from active TB and downregulated with anti-TB treatment [21]. In addition, MPV also served as a clue for the reflection of platelet activation in DM regardless of the diabetic retinopathy stage [25]. In addition to MPV, additional platelet indices PDW and PCT calculated by PC and MPV have been reported to play roles in atherosclerosis and thrombosis, as well in TB [26]. Higher PDW and PCT values developed frequently in patients with PTB with a strong correlation between phase reactants and acute thrombocytosis [27]. However, few studies have explored the relationship between platelet-associated parameters and TBDM coinfection patients. 
The prediction of the risk of TB-DM coinfection is absolutely vital for TB and DM patients. The purpose of the study is to assess the possible relationships of TBDM coinfection with platelet indices PC, MPV, PCT and PDW. To this end, we also determined the relationship of these parameters with inflammatory markers (CRP and ESR index). We propose new novel biomarkers for diabetes with tuberculosis for earlier diagnosis and treatment.

\section{Methods}

\section{Study setting and data sources}

This was a single-centre study. All data were collected in our hospital from July 2018 to August 2019. The study complied with the Declaration of Helsinki, and the Human Ethical Committee of the Sixth People's Hospital of Nantong approved the study protocol. We obtained informed consent from all participants involved in our study. Participants enrolled in the study were given written informed consent. In all, 379 participants were included: 133 healthy controls (HC), $113 \mathrm{~TB}$ patients, 59 DM patients and $74 \mathrm{~TB}+\mathrm{DM}$ patients. $\mathrm{HC}$ were selected by medical examination center with no expose of MTB, no clinical characteristic of TB and the PPD test were negative. TB diagnose based on positive results of Xpert MTB/RIF (Cephaid Inc., CA, USA), BACTEC MGIT 960 rapid liquid isolate culture (Becton Dickinson, Sparks, USA) by GenoTypeH check system (Hain Lifescience, Nehren, Germany) and MTB smear confirmation by Ziehl-Neelsen acid-fast stain (Zhuhai DL biotech co,. Ltd., Guangdong, China). DM patients excluded TB were enrolled from the Endocrine Department in our hospital and diagnosed with $\mathrm{DM}$ previously according to a WHO-criteria. TB-DM coinfection patients were certified TB along with hyperglycemia (fasting glucose $\geq 7.0$ $\mathrm{mmol} / \mathrm{L}$ ) and $\mathrm{HbA1c} \geq 6.5 \%$. Full blood counts were carried out using Mindray BC-6900 chemistry analyzer (Shenzhen Mindray Bio-Medical Electronics Co. Ltd., China). The data of ESR (Erythrocyte sedimentation rate) and CRP (C-reactive protein) were picked from the Clinical Laboratory Department of our hospital measured by Eriline AR Linear (Barcelona, Spain) and Beckman Coulter 5800 (Tokyo, Japan). Participants were obviated if they were positive HIV examination, pregnant, Hepatitis B positive or combined with affecting the platelet indices associated diseases (i.e., cardiovascular disease, hypertension, juvenile systemic lupus erythematous (SLE), Crohn's disease, cancer and others).

\section{Data analysis}

All data processing and analyses were applied using GraphPad Prism version 5.0 software (San Diego, CA) and SPSS 17.0 software (SPSS Inc., Chicago, IL, USA). The difference between unpaired samples was analyzed using one-way ANOVA, $t$-test or chi-squared test. For the basic statistic for the cases enrolled in this study, percentiles and Mean \pm SD were used. The area under the curve (AUC), 95\% confidence interval (95\% CI) sensitivity and specificity were determined by a Receiver Operating Characteristic (ROC) curve. The association between 2 quantitative variables was measured using bivariate correlation (Pearson or Spearman). All tests were two-tailed and a threshold of $P<0.05$ was perceived as statistically significant.

\section{Results}

\section{Characteristics of the study population}

Patient characteristics are shown in Table 1. In the present study, the age ranged from 14 to 90 years. On average, patients with DM $(59.6 \pm 14.0)$ and $\mathrm{TB}+\mathrm{DM}$ $(58.1 \pm 12.5)$ were older and had a lower BMI $(20.0 \pm 1.9$, $20.6 \pm 2.2)$ than the $\mathrm{HC}(45.4 \pm 17.2,20.9 \pm 2.1)$ and $\mathrm{TB}$ $(47.0 \pm 17.8,21.1 \pm 2.4)$ groups. In the present study, the total number of males among the four groups was 204 $(53.83 \%)$, and the total number of females was 175 (46.17\%). The number of males in the TB group was 112 (54.90\%), with $92(45.10 \%)$ in the non-TB group, and the number of females in the TB group was 75 (42.86\%), with 100 in the non-TB group (57.14\%). The were 78 males in the DM group (38.24\%) and $126(61.76 \%)$ in non-TB group and 55 (31.43\%) females in the DM group and $120(68.57 \%)$ in the non-TB group. All (without TB) DM patients were on anti-DM drug treatment, while $67.6 \%$ had TB + DM. TB + DM patients not on any treatment were given a definite diagnosis of DM. In addition, the increased glucose had no effect on interferon- $\gamma$ release, TB drug resistance or TB pulmonary cavity formation (TB vs TB + DM).

\section{MPV and PCT might be new laboratory indicators in TB combined with DM patients}

To investigate whether the platelet influence was involved in $\mathrm{TB}+\mathrm{DM}$ patients, the changes in plateletrelated parameters in the four groups were analyzed by our laboratory. As shown in the results, no differences were found in PC and PDW among the four groups $(P>$ 0.05, Fig. 1a, d, Table 2). Compared with TB $(9.42 \pm 1.01$ $\mathrm{fL})$, the changes in MPV were significantly increased in $\mathrm{TB}+\mathrm{DM}$ patients $(9.95 \pm 1.25 \mathrm{fL}, P=0.0041$, Fig. $1 \mathrm{~b}$, Table 2). Compared with DM patients $(10.92 \pm 1.17 \mathrm{fL}$, $0.22 \pm 0.04 \%)$, the changes in MPV and PCT were significantly decreased in $\mathrm{TB}+\mathrm{DM}$ patients $(9.95 \pm 1.25 \mathrm{fL}$, $0.20 \pm 0.05 \%, P<0.0001, P=0.0121$, Fig. 1b-c, Table 2). ROC curve analysis was used for the MPV and PCT values among $\mathrm{DM}$ patients vs $\mathrm{TB}+\mathrm{DM}$ patients and the MPV value among TB patients vs TB-DM coinfection patients. The sensitivity and specificity of MPV in the differential diagnosis of $\mathrm{DM}$ patients vs $\mathrm{TB}+\mathrm{DM}$ 
Table 1 Patient characteristics in the four groups $(n=379)$

\begin{tabular}{|c|c|c|c|c|c|}
\hline \multirow[t]{2}{*}{ Characteristic } & \multirow{2}{*}{$\begin{array}{l}\mathrm{HC} \\
n=133\end{array}$} & \multirow{2}{*}{$\begin{array}{l}\text { TB } \\
n=113\end{array}$} & \multirow{2}{*}{$\begin{array}{l}\text { DM } \\
n=59\end{array}$} & \multirow{2}{*}{$\begin{array}{l}\text { TB+DM } \\
n=74\end{array}$} & \multirow[t]{2}{*}{$P$ value } \\
\hline & & & & & \\
\hline Sex (male/female) & $60 / 73$ & $66 / 47$ & $32 / 27$ & $46 / 28$ & $0.0497^{*}$ \\
\hline Age (years) & $45.4 \pm 17.2$ & $47.0 \pm 17.8$ & $59.6 \pm 14.0$ & $58.1 \pm 12.5$ & $<0.0001^{*}$ \\
\hline $\mathrm{BMI}\left(\mathrm{kg} / \mathrm{m}^{2}\right)$ & $20.9 \pm 2.1$ & $21.1 \pm 2.4$ & $20.0 \pm 1.9$ & $20.6 \pm 2.2$ & $0.0110^{*}$ \\
\hline HbA1c (\%) & $N A^{a}$ & NA & $11.5 \pm 4.5$ & $11.3 \pm 3.8$ & 0.3915 \\
\hline Fasting glucose & NA & NA & $13.3 \pm 5.5$ & $12.3 \pm 3.7$ & 0.2366 \\
\hline Smoking history & NA & $48 / 113(42.5 \%)$ & 20/59 (33.9\%) & 38/74 (51.4\%) & 0.1219 \\
\hline TSPOT.TB (+) & NA & $96 / 110(87.3 \%)^{b}$ & NA & $42 / 74(56.6 \%)$ & 0.5726 \\
\hline DM medication & NA & NA & $59 / 59(100.0 \%)$ & $50 / 74(67.6 \%)$ & $0.0011^{*}$ \\
\hline Insulin & & & 10/59 (16.9\%) & $5 / 50(10.0 \%)$ & 0.0832 \\
\hline Metformin & & & $42 / 59(71.2 \%)$ & $43 / 50(86.0 \%)$ & 0.5362 \\
\hline Others & & & $7 / 59$ (11.9\%) & $2 / 50(4.0 \%)$ & 0.1591 \\
\hline Years since DM diagnose & NA & NA & & & \\
\hline$<1$ & & & 0/59 (0.0\%) & 11/74 (14.9\%) & $0.0006^{*}$ \\
\hline $1-5$ & & & $21 / 59(35.6 \%)$ & $32 / 74(43.2 \%)$ & $0.0006^{*}$ \\
\hline $6-15$ & & & $28 / 59(47.5 \%)$ & 28/74 (37.8\%) & 0.2695 \\
\hline$>15$ & & & 10/59 (16.9\%) & $3 / 74(4.1 \%)$ & $0.0070^{*}$ \\
\hline With TB drug resistant & NA & 8/113 (7.1\%) & NA & $2 / 74(2.7 \%)$ & 0.1953 \\
\hline With TB pulmonary cavity & NA & $32 / 113(28.3 \%)$ & NA & 15/74(20.0\%) & 0.2169 \\
\hline
\end{tabular}

Different characteristic and statistic results among the four groups. $H C$ Healthy community controls, $T B$ Tuberculosis group, DM Diabetes group, $T B+D M$ TB-DM comorbidity group, BMI Body Mass Index, HbA1c Hemoglobin A1c, TSPOT.TB tuberculosis infectious T-lymphocyte spot assay; ${ }^{a} N A$ not applicable; ${ }^{b}$ Data available from $96 / 110$ patients. *Significant value $(P<0.05)$

patients were defined as 64.9 and $66.1 \%$, respectively (Fig. 1g, Table 3, $P<0.0001$ ), and as 60.8 and $66.4 \%$ in the differential diagnosis of TB patients vs TB + DM patients (Fig. 1h, Table 3, $P=0.003$ ). MPV improved the diagnosis sensitivity when combined with clinical parameters such as fasting blood glucose in DM and $M$. tuberculosis culture result in TB patients $(76.3 \%$ vs 64.9 , $72.6 \%$ vs $60.8 \%$, Fig. 1 -h, Table $3, P<0.0001, P=0.001$, separately). In addition, the sensitivity and specificity of PCT in the differential diagnosis of DM patients vs TB + DM patients were 69.5 and 59.4\%, respectively (Fig. 1i, Table $3, P=0.008)$. PCT improved the diagnosis sensitivity when combined with fasting blood glucose in DM patients $(72.9 \%$ vs $64.9 \%$, Fig. $1 \mathrm{i}$, Table $3, P=0.004)$. ROC-related parameter data are shown in Table 3. Sex and age affected MPV and PCT expression (Fig. 1e-f). Thus, MPV and PCT might be laboratory markers distinguishing $\mathrm{TB}+\mathrm{DM}$ patients from $\mathrm{TB}$ or $\mathrm{DM}$ patients.

\section{MPV is associated with the course of an inflammatory condition instead of PCT}

CRP and ESR are common markers of inflammatory status. The correlation of these markers with MPV and PCT was also analyzed. MPV was associated with ESR $(r=0.3203, P=0.0054$, Fig. $2 \mathrm{a})$ and CRP $(r=0.2504, P=$
0.0307, Fig. $2 \mathrm{~b}$ ) values in the TB + DM group, while it was not associated with CRP and ESR in PCT $(r=0.1905, r=$ $0.008675, P>0.05$, separately, Fig. 2c-d). Thus, MPV might be used as a potential indicator to evaluate whether TB or DM will develop into TB-DM coinfection, and it is correlated to the inflammatory index (CRP and ESR).

\section{Discussion}

The diagnosis of TB has always used a compound method, combining clinical symptoms, etiological examination of MTB, radiology examination and TB-associated molecular testing [28]. The diagnosis of DM is based on globally accepted criteria, relying on fasting plasma glucose measurement, 2-h postchallenge results in an oral glucose tolerance test (OGTT), or a hemoglobin A1c report [29]. Unfortunately, there are no diagnostic standards for DMTB coinfection. The International Diabetes Foundation predicted a prevalence of DM among patients with TB at $8.8 \%$ in 2017 . The global prevalence is almost twice that reported by the International Diabetes Foundation. However, Noubiap concluded that the population may recommend screening for TB patients for diabetes in highincome countries, and in low-income countries, diabetes has a lower prevalence and lower health status and fewer medical resources and screening methods and requires 


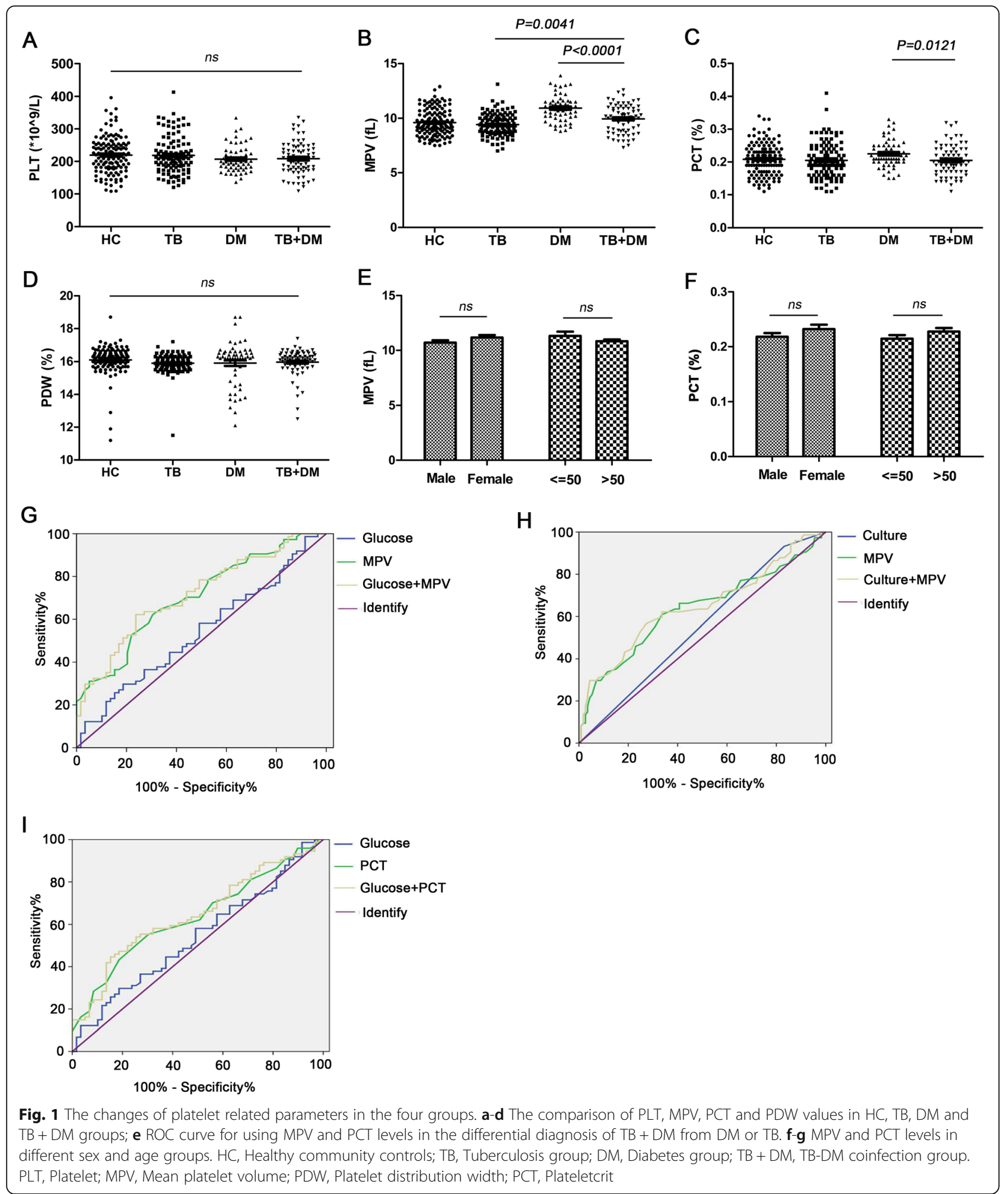

more research to determine the most systematic diagnostic strategies [9].

Platelet indices have been associated with multiple diseases of the immune system and hemopoietic system
[30]. In our study, we found that MPV and PCT were significantly decreased in TB-DM coinfections compared with DM individuals. The indices of MPV were higher in $\mathrm{DM}$ combined with $\mathrm{TB}$ than in $\mathrm{TB}$ patients. The 
Table 2 Comparison of platelet indices among the four groups

\begin{tabular}{llllllll}
\hline & HC & TB & DM & TB+DM & TB vS DM & TB VS TB+DM & $\begin{array}{l}\text { DM VS TB+DM } \\
\boldsymbol{P} \text { value }\end{array}$ \\
\hline $\mathrm{PLT}, \times 10^{9} / \mathrm{L}$ & $219.4 \pm 55.4$ & $218.4 \pm 56.9$ & $207.0 \pm 40.3$ & $208.7 \pm 50.1$ & 0.3050 & 0.1936 & 0.8575 \\
$\mathrm{MPV}, \mathrm{fL}$ & $9.60 \pm 1.21$ & $9.42 \pm 1.01$ & $10.92 \pm 1.17$ & $9.95 \pm 1.25$ & $<0.0001^{*}$ & $0.0041^{*}$ & $<0.0001^{*}$ \\
$\mathrm{PDW}, \%$ & $16.1 \pm 0.8$ & $15.9 \pm 0.6$ & $15.9 \pm 1.3$ & $16.0 \pm 0.8$ & 0.6804 & 0.3821 & 0.3409 \\
$\mathrm{PCT}, \%$ & $0.21 \pm 0.05$ & $0.20 \pm 0.05$ & $0.22 \pm 0.04$ & $0.20 \pm 0.05$ & $0.0397^{*}$ & 0.7066 & $0.0121^{*}$ \\
\hline
\end{tabular}

Platelet associated parameters and statistic results among the four groups. $H C$ Healthy community controls, $T B$ Tuberculosis group, $D M$ Diabetes group, $T B+D M$ TB-DM co-morbidity group, PLT Platelet, MPV Mean platelet volume, $P D W$ Platelet distribution width, $P C T$ Plateletcrit. *Significant value $(P<0.05)$

increase in MPV was correlated with ESR and CRP in $\mathrm{DM}+\mathrm{TB}$ patients. For the PLT and PDW indices, there was no significant change among TB, DM, and TB combined with DM and in healthy controls. Moreover, the factors of age and gender did not significantly affect the MPV and PLT indices.

The MPV reflects platelet size and the extent of inflammation and is used to reveal the function of platelets. The platelet size is associated with the inflammatory intensity [31]. In patients infected by $M$. tuberculosis, acute phase reactants and proinflammatory cytokines affect megakaryocytes, which decrease the platelet size, and smaller platelets are delivered from the bone marrow [32], which can be used to explain the decrease in MPV. Tozkoparan $\mathrm{E}$ et al. found that PDW and PCT were higher in active TB patients and decreased significantly after antituberculosis therapy [21]. Sahin et al. indicated that MPV in active TB patients was identical to that in healthy individuals and nonspecific pneumonia patients [23]. These results were in accordance with our findings, in that there were no significant differences between TB patients and healthy controls. Gunluoglu et al. suggested that the value of MPV was slightly decreased in TB patients, and the MPV never reflected the severity of tuberculosis [24].

ESR has also been regarded as a predictor of inflammatory and autoimmune diseases. In principle, the increase in ESR is due to changes in serum proteins, or it is due to changes in erythrocytes. The former usually includes hypergammaglobulinemia, monoclonal blood diseases, and elevated fibrinogen levels. The latter is mainly to reduce the number of erythrocytes and the size of erythrocytes [33]. Our results showed that MPV, but not PLT, was correlated with ESR in TB patients with DM. Thus, it can be deduced that, as an index of blood in patients with TB and DM, MPV may be an important hematological indicator to evaluate the risk of TB and DM along with ESR.

It is well known that DM is a metabolic dysfunction characterized by hyperglycemia, which leads to vascular

Table 3 The associated parameters of ROC in TB, DM and TB + DM groups

\begin{tabular}{|c|c|c|c|c|c|}
\hline & Sensitivity\% & Specificity $\%$ & AUC & $95 \% \mathrm{Cl}$ & $P$ \\
\hline \multicolumn{6}{|c|}{ DM vs TB+DM group } \\
\hline Glucose & 52.5 & 50.0 & 0.540 & $0.441-0.638$ & 0.432 \\
\hline MPV & 64.9 & 66.1 & 0.705 & $0.618-0.792$ & $<0.0001^{*}$ \\
\hline Glucose+MPV & 76.3 & 62.2 & 0.716 & $0.630-0.802$ & $<0.0001^{*}$ \\
\hline \multicolumn{6}{|c|}{ TB vs TB+DM group } \\
\hline Culture & 17.0 & 93.2 & 0.553 & $0.469-0.636$ & 0.227 \\
\hline MPV & 60.8 & 66.4 & 0.630 & $0.544-0.716$ & $0.003^{*}$ \\
\hline Culture+MPV & 72.6 & 56.8 & 0.645 & $0.561-0.729$ & $0.001^{*}$ \\
\hline \multicolumn{6}{|c|}{ DM vs TB+DM group } \\
\hline Glucose & 52.5 & 50.0 & 0.540 & $0.441-0.638$ & 0.432 \\
\hline PCT & 69.5 & 55.4 & 0.635 & $0.541-0.728$ & $0.008^{*}$ \\
\hline Glucose+PCT & 72.9 & 55.4 & 0.645 & $0.552-0.738$ & $0.004^{*}$ \\
\hline
\end{tabular}

The AUC curve results of MPV and PCT in TB, DM and TB+DM groups. TB Tuberculosis group, DM Diabetes group, TB+DM TB-DM co-morbidity group, Glucose Fasting blood glucose, Culture mycobacterium tuberculosis culture, PLT Platelet, MPV Mean platelet volume, PDW Platelet distribution width, PCT Plateletcrit, AUC Area under curve, $95 \%$ Cl $95 \%$ confidence interval. ${ }^{*}$ Significant value $(P<0.05)$ 


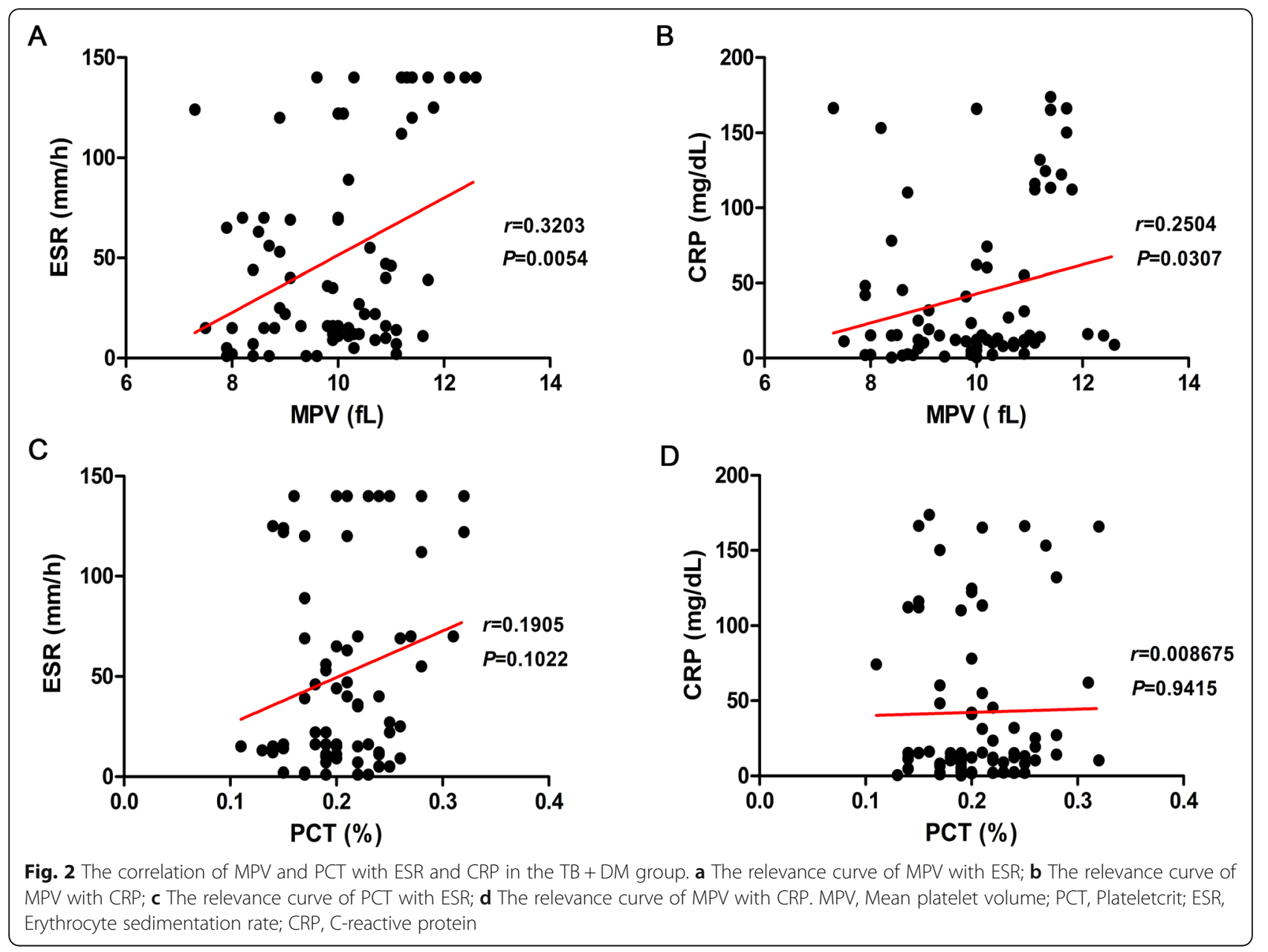

complications. TB is an immemorial and common infectious disease. DM and TB coinfection is a widespread public health issue. There is still a lack of knowledge and assessment regarding whether TB makes individuals susceptible to DM. Several cross-sectional studies have displayed the relationship between $\mathrm{TB}$ outcome and the occurrence of hyperglycemia [34]. As DM and TB increase in low- and middle-income countries, $\mathrm{WHO}$ and the International Union Against Tuberculosis and Lung Disease (IUATLD) encourage the establishment of a cooperative framework that recommends bidirectional screening including $\mathrm{TB}$ testing among individuals with DM [35]. The WHO recommends screening for DM at the start of TB treatment. In China, the bidirectional screening program was implemented in September 2011 [36]. At the time of registration, TB patients were asked if they have diabetes and for those who deny any known disease, a random blood glucose test is performed to determine who is at risk. Patients with high blood glucose levels were randomly followed up with glucose concentration testing. These patients were willing to be screened. A total of 12$13 \%$ of TB patients have diabetes, of which $3 \%$ of Chinese patients are diagnosed with previously unrecognized diabetes based on fasting blood glucose values [8].

A major aim of this study is the comparison of platelet indices (MPV and PCT) and ESR in DM, TB and DM with TB patients to assess whether patients with TB or DM are at risk for developing TB + DM. Screening for TB or DM using platelet indices may improve early TB-DM coinfection detection and diagnosis. This analytical approach meets a clinical goal and establishes a potential diagnosis standard for the clinical laboratory. There is great potential for meaningful research on DM-TB in low- and middle-income regions. Prospective studies are urgently needed to resolve the differences between DM and nonDM tuberculosis patients and TB-DM diagnosis strategies. Identifying the population at risk and then conducting bidirectional screening across the entire region should be the ultimate goal of the health authorities.

\section{Limitations of the study}

Known limitations in this study are that it was a singlecenter study that neglected race and genetic variations. Moreover, there may be a lack of prospective studies 
with a definite diagnosis. To popularize and apply the values of MPV and ESR as diagnostic markers of TB with DM, we should further verify these indices in a multicenter clinical sample and conduct cohort studies in the near future, both in China and abroad. The mechanism of abnormal MPV levels in TB, DM and DM-TB coinfection patients has yet to be fully understood.

\section{Conclusions}

MPV is a valuable candidate marker to screen for TBDM coinfection risk, as the occurrence of TB developing into TB-DM coinfection will increase MPV levels, and DM developing into a DM-TB coinfection will decrease MPV levels. Moreover, MPV has a positive correlation with ESR and CRP. MPV has significant specificity and sensitivity for predicting and diagnosing DM-TB coinfection. Therefore, fasting blood testing (including glucose and MPV) should be carried out in active/ suspected patients with high-risk TB or DM. It is our responsibility to take care of all TB patients, not only in the diagnosis of $\mathrm{TB}$ but also because they are at increased risk of diabetes. Since DM can increase the risk of TB infection and the goal of the WHO strategy to end tuberculosis is to reduce TB deaths by $90 \%$ and TB incidence by $80 \%$ by 2030 , we suggest free treatment policies for DM patients in low- and middle-income countries so that DM and DM-TB coinfection can be better controlled. In conclusion, MPV has potential value as a candidate marker for dual screening algorithms. Screening for diabetic tuberculosis patients should be considered in low-income countries and should be integrated at a culturally educational level and social policy-driven behavior.

\section{Abbreviations}

TB: Tuberculosis; DM: Diabetes mellitus; HC: Healthy control; PC: Platelet count; MPV: Mean platelet volume; PDW: Platelet distribution width; PCT: Plateletcrit; ESR: Erythrocyte sedimentation rate; CRP: C-reactive protein

\section{Acknowledgements}

Not applicable.

\section{Authors' contributions}

YW Q and FF X designed the study; FF X and SY Q collected data; $L W$ and SY Q analyzed and interpreted data; YW Q and FF X drafted the article; FF X and YW Q critically revised the article. All authors approved the final version of the manuscript.

\section{Funding}

The work was supported financially by a grant from China Postdoctoral Science Foundation (2020 M671561), Jiangsu Postdoctoral Science Foundation (2020Z130) and Nantong Science and Technology Program (Grant No. JC2018069) all providing support for the study design and data collection. The project was also supported by the 13th Five-Year Plan Foundation of Nantong and Nantong Junhan business Company (19ZH425) with roles in analysis, publish and preparation of the manuscript.

\section{Availability of data and materials}

The datasets used and analysed during the current study are available from the corresponding author on reasonable request.

\section{Declarations}

\section{Ethics approval and consent to participate}

The study protocol was approved by the Medical Ethics Committee of the Sixth People's Hospital of Nantong. Informed written consent was taken from each participant and this procedure was approved by the Medical Ethics Committee.

\section{Consent for publication}

Not applicable.

\section{Competing interests}

The authors declare that they have no competing interests.

\section{Author details}

'Department of Pathogen Biology, School of Medicine, Nantong University, 19 Qixiu Road, Nantong 226001, Jiangsu, P.R. China. ${ }^{2}$ Department of Clinical Laboratory, The Sixth People's Hospital of Nantong, 500 Yonghe Road, Nantong 226011, Jiangsu, P.R. China. ${ }^{3}$ Department of Cardiothoracic Surgery, Affiliated Hospital of Nantong University, Nantong 226001, Jiangsu, P.R.

China.

Received: 11 January 2020 Accepted: 6 May 2021

Published online: 20 May 2021

\section{References}

1. Dara M, Ehsani S, Mozalevskis A, Vovc E, Simoes D, Avellon Calvo A, et al. Tuberculosis, HIV, and viral hepatitis diagnostics in eastern Europe and Central Asia: high time for integrated and people-centred services. Lancet Infect Dis. 2020;20(2):e47-53. https://doi.org/10.1016/S1473-3099(19)30524-9.

2. Pourostadi M, Rashedi J, Mahdavi Poor B, Samadi Kafil H, Kazemi A, Ahmadpour $E$, et al. Role of molecular epidemiology on tuberculosis control in the Middle East countries: a systematic review and meta-analysis. Tanaffos. 2018;17(4):223-32.

3. Martinez $\mathrm{N}$, Kornfeld $\mathrm{H}$. Tuberculosis and diabetes: from bench to bedside and back. Int J Tuberc Lung Dis. 2019;23(6):669-77. https://doi.org/10.5588/ ijtld.18.0805

4. Pizzol D, Di Gennaro F, Chhaganlal KD, Fabrizio C, Monno L, Putoto G, et al. Tuberculosis and diabetes: current state and future perspectives. Tropical Med Int Health. 2016;21(6):694-702. https://doi.org/10.1111/tmi.12704.

5. Pizzol D, Di Gennaro F, Chhaganlal KD, Fabrizio C, Monno L, Putoto G, et al. Prevalence of diabetes mellitus in newly diagnosed pulmonary tuberculosis in Beira, Mozambique. Afr Health Sci. 2017;17(3):773-9. https://doi.org/1 0.4314/ahs.v17i3.20.

6. McMurry HS, Mendenhall E, Rajendrakumar A, Nambiar L, Satyanarayana S, Shivashankar R. Coprevalence of type 2 diabetes mellitus and tuberculosis in low-income and middle-income countries: a systematic review. Diabetes Metab Res Rev. 2019:35(1):e3066. https://doi.org/10.1002/dmrr.3066.

7. Li Y, Teng D, Shi X, Qin G, Qin Y, Quan H, et al. Prevalence of diabetes recorded in mainland China using 2018 diagnostic criteria from the American Diabetes Association: national cross sectional study. BMJ. 2020; 369:m997.

8. Lonnroth K, Roglic G, Harries AD. Improving tuberculosis prevention and care through addressing the global diabetes epidemic: from evidence to policy and practice. Lancet Diabetes Endocrinol. 2014;2(9):730-9. https://doi. org/10.1016/S2213-8587(14)70109-3.

9. Noubiap JJ, Nansseu JR, Nyaga UF, Nkeck JR, Endomba FT, Kaze AD, et al. Global prevalence of diabetes in active tuberculosis: a systematic review and meta-analysis of data from 2.3 million patients with tuberculosis. Lancet Glob Health. 2019;7(4):e448-60. https://doi.org/10.1016/S2214-109X(18)304 87-X.

10. Dooley KE, Chaisson RE. Tuberculosis and diabetes mellitus: convergence of two epidemics. Lancet Infect Dis. 2009;9(12):737-46. https://doi.org/10.1016/ S1473-3099(09)70282-8.

11. Alisjahbana B, Sahiratmadja E, Nelwan EJ, Purwa AM, Ahmad Y, Ottenhoff $\mathrm{TH}$, et al. The effect of type 2 diabetes mellitus on the presentation and treatment response of pulmonary tuberculosis. Clin Infect Dis. 2007:45(4): 428-35. https://doi.org/10.1086/519841.

12. Niazi AK, Kalra S. Diabetes and tuberculosis: a review of the role of optimal glycemic control. J Diabetes Metab Disord. 2012;11(1):28. https://doi.org/1 0.1186/2251-6581-11-28. 
13. Lee PH, Fu H, Lai TC, Chiang CY, Chan CC, Lin HH. Glycemic control and the risk of tuberculosis: a cohort study. PLoS Med. 2016;13(8):e1002072. https://doi.org/10.1371/journal.pmed.1002072.

14. Pealing L, Wing K, Mathur R, Prieto-Merino D, Smeeth L, Moore DA. Risk of tuberculosis in patients with diabetes: population based cohort study using the UK clinical practice research Datalink. BMC Med. 2015;13(1):135. https://doi.org/10.1186/s12916-015-0381-9.

15. Leegaard A, Riis A, Kornum JB, Prahl JB, Thomsen VO, Sorensen HT, et al Diabetes, glycemic control, and risk of tuberculosis: a population-based case-control study. Diabetes Care. 2011;34(12):2530-5. https://doi.org/10.233 7/dc11-0902.

16. Awad SF, Huangfu P, Ayoub HH, Pearson F, Dargham SR, Critchley JA, et al. Forecasting the impact of diabetes mellitus on tuberculosis disease incidence and mortality in India. J Glob Health. 2019;9(2):020415. https://doi, org/10.7189/jogh.09.020415.

17. Pizzol D, Veronese N, Marotta C, Di Gennaro F, Moiane J, Chhaganlal K, et al. Predictors of therapy failure in newly diagnosed pulmonary tuberculosis cases in Beira, Mozambique. BMC Res Notes. 2018;11(1):99. https://doi.org/1 0.1186/s13104-018-3209-9.

18. Margraf A, Zarbock A. Platelets in inflammation and resolution. J Immunol. 2019;203(9):2357-67. https://doi.org/10.4049/jimmunol.1900899.

19. Korniluk A, Koper-Lenkiewicz OM, Kaminska J, Kemona H, Dymicka-Piekarska $\checkmark$. Mean platelet volume (MPV): new perspectives for an old marker in the course and prognosis of inflammatory conditions. Mediat Inflamm. 2019; 2019:9213074.

20. Farias MG, Schunck EG, Dal Bo S, de Castro SM. Definition of reference ranges for the platelet distribution width (PDW): a local need. Clin Chem Lab Med. 2010:48:255-7.21.

21. Tozkoparan E, Deniz O, Ucar E, Bilgic H, Ekiz K. Changes in platelet count and indices in pulmonary tuberculosis. Clin Chem Lab Med. 2007;45(8): 1009-13. https://doi.org/10.1515/CCLM.2007.194.

22. Lee MY, Kim YJ, Lee HJ, Cho SY, Park TS. Mean platelet volume in mycobacterium tuberculosis infection. Biomed Res Int. 2016;2016:7508763.

23. Sahin F, Yazar E, Yildiz P. Prominent features of platelet count, plateletcrit, mean platelet volume and platelet distribution width in pulmonary tuberculosis. Multidiscip Respir Med. 2012;7(1):38. https://doi.org/10.1186/2 049-6958-7-38.

24. Gunluoglu G, Yazar EE, Veske NS, Seyhan EC, Altin S. Mean platelet volume as an inflammation marker in active pulmonary tuberculosis. Multidiscip Respir Med. 2014;9(1):11. https://doi.org/10.1186/2049-6958-9-11.

25. Citirik M, Beyazyildiz E, Simsek M, Beyazyildiz O, Haznedaroglu IC. MPV may reflect subcinical platelet activation in diabetic patients with and without diabetic retinopathy. Eye (Lond). 2015;29(3):376-9. https://doi.org/10.1038/ eye.2014.298.

26. Singh A, Varma R. Role of platelet distribution width (PDW) and Plateletcrit in the assessment of nonthrombocytopenic preeclampsia and Eclampsia. Obstet Gynaecol India. 2018;68(4):289-93. https://doi.org/10.1007/s13224-01 7-1036-x.

27. Ding RD, Zhang HJ. Effect of linezolid on serum PCT, ESR, and CRP in patients with pulmonary tuberculosis and pneumonia. Medicine (Baltimore). 2018;97(37):e12177. https://doi.org/10.1097/MD.0000000000012177.

28. Pai M, Behr MA, Dowdy D, Dheda K, Divangahi M, Boehme CC, et al Tuberculosis. Nat Rev Dis Primers. 2016;2(1):16076. https://doi.org/10.1038/ nrdp.2016.76.

29. Reddy SSK. Diagnosis of diabetes mellitus in older adults. Clin Geriatr Med. 2020;36(3):379-84. https://doi.org/10.1016/j.cger.2020.04.011.

30. Mohamed AB, Elnady HM, Alhewaig HK, Moslem Hefny H, Khodery A. The mean platelet volume and plateletcrit as predictors of short-term outcome of acute ischemic stroke. Egypt J Neurol Psychiatr Neurosurg. 2019;55(1):4. https://doi.org/10.1186/s41983-018-0035-x.

31. Gasparyan AY, Ayvazyan L, Mikhailidis DP, Kitas GD. Mean platelet volume: a link between thrombosis and inflammation? Curr Pharm Des. 2011;17(1):4758. https://doi.org/10.2174/138161211795049804.

32. Bath PM, Butterworth RJ. Platelet size: measurement, physiology and vascular disease. Blood Coagul Fibrinolysis. 1996;7(2):157-61. https://doi. org/10.1097/00001721-199603000-00011.

33. Lapic I, Padoan A, Bozzato D, Plebani M. Erythrocyte sedimentation rate and C-reactive protein in acute inflammation. Am J Clin Pathol. 2019. https://doi. org/10.1093/ajcp/aqz142.
34. Bailey SL, Ayles H, Beyers N, Godfrey-Faussett P, Muyoyeta M, du Toit E, et al. The association of hyperglycaemia with prevalent tuberculosis: a population-based cross-sectional study. BMC Infect Dis. 2016;16(1):733. https://doi.org/10.1186/s12879-016-2066-1.

35. Billo N, Castro JL, Jones S, Rusen ID, Chiang CY, Fussell M, et al. The International Union against Tuberculosis and Lung Disease: past, present and future. Int Health. 2009; 1(2):117-23. https://doi.org/10.1016/j.inhe.2009.09.001.

36. Lin Y, Li L, Mi F, Du J, Dong Y, Li Z, et al. Screening patients with diabetes mellitus for tuberculosis in China. Tropical Med Int Health. 2012;17(10):13028. https://doi.org/10.1111/j.1365-3156.2012.03069.x.

\section{Publisher's Note}

Springer Nature remains neutral with regard to jurisdictional claims in published maps and institutional affiliations.
Ready to submit your research? Choose BMC and benefit from:

- fast, convenient online submission

- thorough peer review by experienced researchers in your field

- rapid publication on acceptance

- support for research data, including large and complex data types

- gold Open Access which fosters wider collaboration and increased citations

- maximum visibility for your research: over $100 \mathrm{M}$ website views per year

At BMC, research is always in progress.

Learn more biomedcentral.com/submissions 